\title{
Tsafon
}

Revue d'études juives du Nord

$75 \mid 2018$

Exil des langues - Langues d'exil

\section{L'exil de la langue. Le poète Günter Kunert entre Est et Ouest}

\section{Martine Benoit}

\section{(2) OpenEdition}

12 Journals

Édition électronique

URL : https://journals.openedition.org/tsafon/588

DOI : $10.4000 /$ tsafon.588

ISSN : 2609-6420

Éditeur

Association Jean-Marie Delmaire

Édition imprimée

Date de publication : 1 juin 2018

Pagination : 81-95

ISSN : 1149-6630

\section{Référence électronique}

Martine Benoit, «L'exil de la langue. Le poète Günter Kunert entre Est et Ouest », Tsafon [En ligne], 75 | 2018, mis en ligne le 06 mai 2019, consulté le 24 juin 2021. URL : http://journals.openedition.org/ tsafon/588; DOl : https://doi.org/10.4000/tsafon.588

Tsafon. Revues d'études juives du Nord 


\title{
L'exil de la langue Le poète Günter Kunert entre Est et Ouest
}

\author{
Martine Benoit*
}

Günter Kunert est considéré en Allemagne comme un des écrivains dissidents les plus intègres et les plus courageux de l'ancienne RDA - un des plus brillants aussi. Né à Berlin le 6 mars 1929, il est classé selon les lois raciales édictées en 1935 à Nuremberg " Mischling ersten Grades », [métis au premier degré], sa mère, Edith Kunert, née Warschauer, étant considérée comme de "race juive » : tous deux doivent leur survie à leur père et mari qui refusa de divorcer et de les abandonner ${ }^{1}$. À la fin de la guerre, la famille Kunert décide de rester en zone d'occupation soviétique, Günter Kunert fait des études d'art à la Hochschule für angewandte Kunst [Université d'art appliqué] à Berlin-Weißensee, travaille pour différentes revues, notamment la revue satirique Ulenspiegel. Dans ses débuts poétiques, Kunert est remarqué puis appuyé par Johannes R. Becher (1891-1958) ${ }^{2}$. Kunert publie son premier recueil de poèmes en RDA en 1950 sous le titre Wegschilder und Mauerinschriften [Panneaux indicateurs et inscriptions murales], et en

\footnotetext{
${ }^{*}$ Université de Lille, SHS.

${ }^{1}$ Sur la judéité de Günter Kunert voir : Martine Benoit, « ‘... et c’est devenu mon destin' - Günter Kunert et la judéité », dans Marie-Hélène Quéval (coord.), Lectures d'une œuvre - Günter Kunert, Paris, Éditions du Temps, 2000, p. 73-90.

${ }^{2}$ Ancien poète expressionniste qui avait rejoint les communistes dès 1919, Johannes R. Becher s'était exilé à Moscou pendant la guerre pour revenir aux premières heures de la défaite allemande et devenir en juillet 1945 le premier Président du «Kulturbund zur demokratischen Erneuerung Deutschlands» [Ligue culturelle pour le renouveau démocratique de l'Allemagne], puis en 1954 le premier ministre de la culture de RDA.
} 
RFA en 1963, Erinnerungen an einen Planeten [Souvenirs d'une planète]. Cependant, son intégrité et son exigence de vérité font rapidement de lui un auteur rétif et encombrant pour Berlin-Est. La rupture est complète en 1976 lors de la co-écriture et de la signature de la pétition soutenant le chanteur Wolf Biermann, interdit de retour à l'Est alors qu'il donne un concert à Cologne. Trois années plus tard, Günter Kunert et sa femme Marianne s'installent définitivement en RFA.

Dans un précédent article consacré à la notion d'exil chez Günter Kunert $^{3}$, j'ai analysé comment celui-ci avait interrogé son départ de RDA, son arrivée en RFA, son lien à la RDA, à la RFA, puis à l'Allemagne réunifiée. Ce qui ressort de cette analyse, c'est un sentiment profond de déracinement, une impossibilité intérieure de se vivre Allemand, une absence d'identification charnelle à un pays quelconque, que ce soit la RFA, la RDA ou l'Allemagne. Au cœur de cette analyse, il y avait la conviction de Günter Kunert que tout sentiment national d'attachement était immanquablement perverti :

Il reste qu'en Allemagne, derrière tout signe national, apparaissent les ombres des fours crématoires: chaque nouvelle exploitation d'éléments nationaux rappelle immanquablement les crimes car ceux-ci ont été perpétrés au nom de la Nation, sous son sceau et prétendument pour son bien. ${ }^{4}$

Pour Günter Kunert, la seule Heimat possible est imaginaire, la seule Heimat possible est celle de l'écriture : la littérature est le seul lieu de la reconstruction d'une identité possible après Auschwitz.

C'est de cette recherche et de ce constat que je voudrais partir pour analyser ce que signifie pour Günter Kunert écrire en allemand, pour voir comment l'écrivain investit la langue allemande, quel est son travail sur cette langue, comment il appréhende la littérature et son rôle comprendre ce que signifie pour Günter Kunert être un poète de langue allemande d'origine juive dont la vie et l'œuvre témoignent de ce

\footnotetext{
${ }^{3}$ Voir Martine Benoit, «'Fremd daheim' - étranger chez soi - le sentiment d'exil chez Günter Kunert », Tsafon - Revue d'études juives du Nord, n49, printemps-été 2005, p. 85-98.

${ }^{4}$ Günter Kunert, « Ungemeinsame Geschichte - erinnern und weiterleben », dans Die letzten Indianer Europas: Kommentare zum Traum, der Leben heißt, München, Hanser, 1991, p. 222-235 (ici p. 234) : "Prekär bleibt, daß in Deutschland hinter allen Kennzeichen des Nationalen die Schatten der Krematorien deutlich sind, daß jeder Wiedergebrauch nationaler Elemente notwendigerweise an die Verbrechen erinnert, da diese namens der Nation und zu ihrem vorgeblichen Nutzen und unter ihrem Siegel begangen wurden ». Toutes les traductions des textes de Günter Kunert sont de moi.
} 
qu' « après Auschwitz, nul ne peut écrire qu'en vertu d'Auschwitz » ${ }^{5}$. Je voudrais montrer ici un double mouvement dans l'écriture de Günter Kunert: son désir de s'inscrire dans une tradition littéraire allemande dont il interroge les codes d'écriture et les compromissions; son attention permanente à inscrire au cœur de son écriture la mise en garde et d'affirmer la poésie comme acte de résistance à l'histoire et à l'époque.

\section{S'inscrire dans la tradition littéraire allemande en l'interrogeant}

Günter Kunert se montre désireux de reprendre à son compte, en les détournant et en les dévoyant, des éléments de la tradition allemande. Il y a notamment chez cet écrivain un goût pour la réécriture des contes allemands, des contes de Grimm. Günter Kunert publie ainsi en 1973 une version de La Belle au Bois dormant ${ }^{6}$, en 1988 une version contemporaine d'Hansel et Gretel'. Le conte revisité dévoile les éléments menaçants qui étaient certes présents dans les contes traditionnels mais qui étaient dénoués à la fin de l'histoire : malgré son sommeil de cent années, la Belle reste belle ; Hansel et Gretel de leurs côtés se perdent certes dans la forêt, trouvent refuge chez une vieille sorcière mais retrouvent, à la fin, la maison de leur père pour y vivre en paix avec l'or de la vilaine femme. Chez Günter Kunert, le conte préserve jusqu'au bout ses aspects menaçants : la Belle est une «vieille rombière » disgracieuse à son réveil ; le protagoniste de «Perdu en chemin » est seul, la maison qu'il trouve au milieu de la forêt est en ruine, il ne la détruit donc pas (à l'inverse de Hansel et Gretel qui détruisent une partie de la maison en la mangeant) - contrairement à Hansel et Gretel qui font brûler la sorcière et se libèrent, le personnage de Günter Kunert reste dans la maison détruite, ne trouve pas d'issue ni de libération. Günter Kunert congédie la bonté et le courage et abandonne son lecteur et ses personnages à leur triste destin.

Günter Kunert s'inscrit également dans la tradition de la littérature fantastique allemande, exploitant la double possibilité de l'écriture

\footnotetext{
${ }^{5}$ Expression empruntée à l'article de Jacques Ascher, « 'Ceux qui disent la vérité disent les ombres' », dans Bruno Gravier, Jean-Marc Elchardus, Marcel Colin, Le crime contre l'humanité, Toulouse, éditions, érès, 1996, p. 155-162 (ici p. 159).

${ }^{6}$ Voir la nouvelle « Dornröschen », dans Die geheime Bibliothek, Berlin und Weimar, Aufbau, 1973.

${ }^{7}$ Il s'agit ici de la nouvelle "Auf Abwegen» [Perdu en chemin»] du recueil Auf Abwegen und andere Verirrungen, München, Hanser, 1988.
} 
fantastique. Certaines nouvelles intègrent ainsi dans une histoire quotidienne un élément étranger, inconnu, comme l'«Histoire quotidienne d'une rue berlinoise ${ }^{8}$ qui raconte la vie de David Platzker, Juif allemand contraint à l'exil, qui part en emportant, enroulée sous son bras, sa rue berlinoise remplie de ses habitants. De retour à Berlin avec la fin de la guerre et l'écroulement du Reich hitlérien, David Platzker retrouve l'emplacement de sa rue et la déroule pour qu'elle reprenne sa place et repart. Pris de remords après une nuit sans sommeil, il revient le lendemain, cherche avec difficulté à retrouver sa rue - elle a disparu : d'elle ne reste qu'une vieille cafetière émaillée au sol d'où s'échappe un liquide rouge qui suggère l'assassinat des Juifs d'Europe et dit l'impossible retour des Juifs en Allemagne et l'impossible réconciliation entre Juifs et Allemands après la Shoah. Dans une autre nouvelle qui paraît elle aussi pour la première fois en 1968, un homme aperçoit lors de son «Voyage en tram », entre deux stations, une fenêtre éclairée qui s'ouvre sur une pièce où il se voit, entouré de ses amis et de ses proches dont « la plupart avaient disparu depuis, avaient été brûlés ou assassinés ou s'étaient exilés ou avaient vieilli $»{ }^{9}$. Chaque semaine, l'homme reprend le métro pour revivre cette scène, sans jamais arriver à pénétrer dans la pièce : le monde d'avant la Catastrophe, le Berlin insouciant de l'enfance, ont disparu à jamais.

Renouant avec la tradition fantastique allemande, Günter Kunert montre, inversement, un monde étranger et inconnu confronté aux problèmes $\mathrm{du}$ quotidien: ainsi la nouvelle «Andromède à contretemps $»^{10}$, située au $\mathrm{XXX}^{\mathrm{e}}$ siècle de notre ère alors que la nourriture manque sur terre et que des transferts de population vers d'autres planètes sont organisés et retransmis à la télévision. Le soir du

\footnotetext{
${ }^{8}$ «Alltägliche Geschichte einer Berliner Straße » est une nouvelle qui a une place un peu à part dans l'œuvre novellistique de Kunert tant elle accompagne la production de cet auteur depuis sa première édition. Cette nouvelle est en effet parue pour la première fois en 1968 à l'Est (dans le recueil Kramen in Fächern - Geschichten. Parabeln. Merkmale, Berlin, Aufbau-Verlag) et à l'Ouest (dans le recueil Die Beerdigung findet in aller Stille statt, München, Hanser) sous le titre «Straßengeschichte » [Histoire d'une rue], puis fut rééditée à de nombreuses reprises, notamment à l'Est en 1979 avec deux autres nouvelles et des gravures de Kunert lui-même sous le titre Drei Berliner Geschichten, ou bien dans Auf Abwegen und andere Verirrungen en 1988, et a été reprise aujourd'hui encore, en 2006, dans le volume Irrtum ausgeschlossen, München, Hanser.

${ }^{9}$ Günter Kunert, « Fahrt mit der S-Bahn », dans Kramen in Fächern. Geschichten Parabeln - Merkmale, Berlin und Weimar, Aufbau, 1968, p. 62-69 (ici p. 64).

${ }^{10}$ "Andromeda zur Unzeit», nouvelle parue en 1968 dans le recueil Die Beerdigung findet in aller Stille statt et fut rééditée dans Auf Abwegen und andere Verirrungen.
} 
10 avril 3000, une chose étrange arrive, les constellations ne sont pas celles d'un mois d'avril mais d'un mois de novembre ; celui qui en fait le constat et qui dit à ses proches et ses amis son trouble, reçoit trois jours plus tard une carte verte annonçant son départ. De nombreux détails de la nouvelle rappellent l'extermination des Juifs d'Europe par les nazis : les colonnes d'hommes et de femmes traînant leur bagage avant de monter dans l'immense fusée censée les mener sur une autre planète ; le ciel de novembre en plein mois d'avril suggère un lien entre le boycott d'avril 1933 et les pogromes de novembre 1938. Il y a aussi cette étrange coïncidence entre la visite aux amis et la réception de la carte verte, qui suppose une dénonciation ou une participation plus active encore. Et la destination aussi est étrange, la constellation de Cassiopée, dans l'hémisphère boréal, rappelant l'attirance pour la pureté nordique. Tout ici respire le camouflage de l'assassinat et évoque le secret autour de ce que les nazis nommèrent, par un euphémisme, la «solution finale de la question juive $»$.

\section{Le travail sur la langue}

Le travail sur la langue est aussi caractéristique de cette double contrainte du poète de langue allemande d'origine juive qui écrit après l'extermination. C'est en effet d'abord dans sa poésie que Günter Kunert tente de réinvestir la langue allemande contaminée, témoin de l'ébranlement causé par Auschwitz. Jacques Derrida utilisait pour Paul Celan l'image de la cicatrice, écrivant que «Paul Celan touche à la langue allemande à la fois dans le respect du génie idiomatique de la langue allemande mais aussi au sens où il la fait bouger, où il y laisse une sorte de cicatrice, de marque, de blessure $»^{11}$. Pendant longtemps, Günter Kunert a gardé la rime allemande, cette rime que Celan avait congédiée, la disloquant par des enjambements, achevant ses phrases au milieu d'un vers, apostrophant sa mère dans le poème «Nähe der Gräber » [Proximité des tombes] et rejetant la rime dans la nostalgie impossible :

Et tu acceptais, mère, comme avant, chez nous,

La rime douce, la rime allemande, la rime douloureuse ${ }^{12}$

\footnotetext{
${ }^{11}$ Interview de Jacques Derrida dans la revue Europe, janvier-février 2001, p. 84.

12 Paul Celan, «Nähe der Gräber», dans P. Celan, Gesammelte Werke in sieben Bänden, Band 6 - Das Frühwerk, Frankfurt/Main, Surkamp, 2000, p. 136 : «Und duldest du, Mutter, wie einst, ach, daheim, / den leisen, den deutschen, den schmerzlichen Reim ?».
} 
On pense aussi à ce rare texte autobiographique de la poétesse de Czernowitz, Rose Ausländer, où un passage aborde ce questionnement sur la rime :

Ce qui plus tard s'abattit sur nous était si absurde, si cauchemardesque, si oppressant que, après le contrecoup, dans le choc entièrement ressenti par la suite, la rime se brisa. Les mots fleuris se flétrirent. Beaucoup d'adjectifs aussi étaient devenus suspects [...]. L'ancien vocabulaire devait être changé. Les étoiles - je n'ai pas pu les éloigner de ma poésie d'Après-guerre - apparurent sous de nouvelles constellations. ${ }^{13}$

Günter Kunert continue pendant un temps à travailler la rime finale, à utiliser l'ancienne rime intérieure (appelée Stabreim en allemand), à se jouer des assonances et des allitérations. Puis c'est par l'abandon de la ponctuation que vient peu à peu le renoncement à la rime, Kunert refusant tout élément d'embellissement. Ainsi dans son poème « Bekenntnis » [Confession] :

\author{
Beaux ? Je ne peux \\ pas écrire de beaux poèmes. \\ Des morts, expulsés de leur vie dans la laideur \\ posent un poids sur ma main. Les mots \\ deviennent litigieux au lieu d'être justes. ${ }^{14}$
}

Pour Günter Kunert, la littérature et plus spécifiquement la poésie ne sont plus le lieu de l'esthétisme et de la beauté, ce sont des lieux de résistance et de mise en garde. En 1972, le poète définissait l'acte d'écriture comme « un geste de libération de soi, de compréhension de soi, de réalisation de soi », un acte d'individuation réussie ${ }^{15}$. Dans ses

13 Rose Ausländer, «Alles kann Motiv sein », dans R. Ausländer, Gedichte, Frankfurt/Main, Fischer, 2005, p. 7-12 (ici page 9) : « Was später über uns hereinbrach, war ungereimt, so alpdruckhaft beklemmend, dass - erst in der Nachwirkung, im nachträglich voll erlittenen Schock - der Reim in die Brüche ging. Blumenworte welkten. Auch viele Eigenschaftsworte waren fragwürdig geworden [...]. Das alte Vokabular musste ausgewechselt werden. Die Sterne - ich konnte sie auch aus meiner Nachkriegslyrik nicht entfernen - erschienen in anderer Konstellation ».

${ }^{14}$ Günter Kunert, «Bekenntnis », dans Fremd daheim. Gedichte, München, Hanser, 1990 , p. 98 : «Schön ? Ich kann / keine schönen Gedichte schreiben. Tote / unschön vertrieben aus ihrem Leben / legen ein Gewicht / auf meine Hand. Die Worte / werden strittg statt trefflich ».

${ }^{15}$ Günter Kunert, «Manche, einige und sogenannte », dans Sinn und Form (1972), p. 110: «Der Akt des Schreibens ist einer der Selbstbefreiung, des 
conférences poétologiques tenues à Francfort en 1982, Kunert définit la poésie comme une « arche de Noé », une possibilité de sauvetage avant le déluge ${ }^{16}$.

\section{La poésie comme lieu de résistance}

C'est justement par la parution d'un recueil de poèmes que Kunert va, pour la première fois, prendre ses distances avec le régime communiste de Berlin-Est et vis-à-vis de la perception téléologique de l'histoire. Il s'agit du recueil au titre significatif Der ungebetene Gast [L'hôte indésirable], recueil qui, bien qu'achevé dès la fin de l'année 1962, ne fut autorisé à la publication qu'en 1965. Tout le recueil marque une rupture dans la production littéraire de Günter Kunert, un véritable renversement des valeurs que le poète semblait considérer jusqu'alors comme établies. Certains critiques parlent de «révolution copernicienne », de «changement de paradigme ${ }^{17}$. L'auteur prévient ses lecteurs dès la jaquette de son recueil: ses poèmes se veulent des mises en garde contre la «déshumanisation, la perte, possible et irrémédiable, de la conditio humana $\|^{18}$; ce qu'il faut, c'est « rendre la terre habitable pour / les hommes ${ }^{19}$. Le processus historique ne mène plus aux lendemains qui chantent mais à la catastrophe. Le voyage vers l'avenir ne mène plus vers l'avant :

Laisse-nous voyager avec

La certitude : Où que nous arrivions

Le but est déjà

Derrière nous. $^{20}$

L'homme n'est plus porteur d'espoir, c'est un opportuniste, dominé par des sentiments tels que le conformisme, l'habitude, l'oubli, le

Selbstverständnisses, der Selbstverwirklichung, einer der einzig möglichen Individuation, nämlich der geglückten Identitätsstiftung ».

${ }^{16}$ Günter Kunert, Vor der Sintflut - das Gedicht als Arche Noah - Frankfurter Vorlesungen, München, Hanser Verlag, 1985.

${ }^{17}$ Voir Elke Kasper, Zwischen Utopie und Apokalypse - das lyrische Werk Günter Kunerts von 1950 bis 1987, Tübingen, Max Niemeyer Verlag, 1995, p. 51.

${ }^{18}$ Günter Kunert, Der ungebetene Gast, Berlin, Aufbau-Verlag, 1965 (texte de la jaquette : «Gezielt ist aber auf die Gefahr der Entmenschlichung, auf den möglichen, unwiederbringlichen Verlust der Conditio humana »).

${ }^{19}$ Ibid., « Vom Vergehen », p. 9 : « Die Erde bewohnbar zu machen für / Menschen ».

${ }^{20}$ Ibid., «Lass uns reisen », p. 41 : «Lass uns reisen in / Gewissheit: Wo wir auch anlangen, / Liegt das Ziel / Schon hinter uns ». 
refoulement. Le poète met inlassablement en garde contre le passé et la vérité refoulés, qui, toujours, refont surface.

Ce qui est remarquable, c'est de constater que ce recueil est le premier à introduire dans la poésie de Günter Kunert le thème de l'extermination et de la mémoire de la Shoah. D'emblée, le titre de l'ouvrage, L'hôte indésirable, annonce ce contexte: dans le discours antisémite en effet, le terme de "Gast » [hôte] est porteur d'une forte charge dépréciatrice.

Dans son ouvrage consacré à La question juive, Alex Bein fait remonter à Luther la perception du peuple juif comme un peuple qui s'invite chez les autres peuples, dans son essai Des Juifs et de leurs mensonges [Von den Juden und ihren Lügen] de $1543^{21}$; on retrouve plus tard cette idée dans La France juive d'Édouard Drumont mais aussi dans les écrits de Heinrich von Treitschke, où le Juif est présenté comme l'« hôte » du peuple allemand, un hôte tout juste toléré [geduldet]. Et on ne s'étonnera pas de trouver dans le programme du parti nazi, le NSDAP, la notion de « Gast » pour nommer l'étranger ${ }^{22}$. Le titre de Günter Kunert accole de plus à ce terme stigmatisant un adjectif marquant un autre refus, «ungebeten » [indésirable] : avant même d'ouvrir l'ouvrage, le lecteur est mis en garde. Le poème éponyme est le premier poème et premier écrit de Kunert à placer au cœur du propos l'extermination des Juifs ou, pour être plus juste, la question du refus de la prise de conscience du crime :

1 Stellt noch einen Stuhl an den Tisch

2 Es ist ein Gast gekommen

(Aus der Gegend um Warschau dort)

Und hat am Tisch Platz genommen

Und sagte kein einziges Wort

3 Füllet ihm ein Glas

\footnotetext{
${ }^{21}$ Voir Alex Bein, Die Judenfrage, Stuttgart, DVA, 1980. Pour le texte de Luther, se reporter à l'excellente dernière édition de Matthias Morgenstern : Martin Luther, Von den Juden und ihren Lügen - neu bearbeitet und kommentiert von Matthias Morgenstern, Wiesbaden, Berlin University Press, 2016 (édition recensée dans Germanica $\mathrm{n}^{\circ}$ 59/2016, en ligne : https://journals.openedition.org/germanica/3584).

${ }^{22}$ Point 5 du programme en 25 points du NSDAP : « Wer nicht Staatsbürger ist, soll nur als Gast in Deutschland leben können und muss unter Fremdengesetzgebung stehen », cité dans Walther Hofer (éd.), Der Nationalsozialismus - Dokumente 1933-1945, Frankfurt/Main, Fischer 1957, p. 28.
} 
4 Die Füße mit Lappen umwunden,

Und die Augen haben gefehlt.

An der Kehle klaffende Wunden

Haben stumm seine Geschichte erzählt.

5 Was steht dem Gast zu Diensten?

6 Er schwieg gleich der dunklen Tiefe

Im allertiefsten Meer.

Dann hob er den Kopf, als riefe

Seinen Namen irgendwer.

7 Öffnet ihm die Tür.

8 So ist der Gast gegangen

Sacht wie ein Licht verlischt.

Sich doch zum Essen zwangen,

Denen man aufgetischt.

9 Wohl bekomm es.

10 Da schmeckten nach Asche die Bissen, und die Esser senkten den Blick;

Voreinander ihre Gewissen

Verbargen sie ohne Geschick.

11 So rückt doch den Stuhl wieder fort.

[1 Ajoutez une chaise à table ; 2 Il est venu un hôte / (De la région de Varsovie là-bas) / Qui a pris place à table / Et n'a prononcé aucun mot ; 3 Remplissez-lui un verre; 4 Les pieds entourés de chiffons / Et les yeux manquaient. / À la gorge des plaies ouvertes / Disaient sans mot son histoire; 5 Que proposez-vous à l'hôte ?; 6 Il se taisait, semblable à la profondeur obscure / De la plus profonde des mers / Puis il leva la tête comme si / Quelqu'un appelait son nom ; 7 Ouvrezlui la porte ; 8 C'est ainsi que l'hôte est parti / Aussi doucement que s'éteint une bougie / Ceux qui étaient à table / Se forcèrent à manger; 9 À votre santé ; 10 Les bouchées avaient un goût de cendre / Et les convives baissèrent le regard ; / Se cachant sans habileté / Leur conscience. ; 11 Enlevez donc la chaise].

Trois groupes sont présents dans le poème: "der Gast » [L'invité]; un groupe d'hommes, «die Esser» [les mangeurs]; un narrateur qui intervient par des injonctions. L'invité «de la région de Varsovie là-bas », allusion à Auschwitz, entre dans une maison où des gens sont attablés. Élie indésirable, que personne n'attend, ce revenant aux blessures béantes, aux yeux vides, aux habits en lambeaux, se tait, puis repart : il n'est plus le prophète annonciateur des temps rédempteurs. 
Les «mangeurs» baissent la tête et font taire leur conscience : est suggéré ici le refus de la confrontation avec les crimes commis; le silence se fait accusateur devant tant de refoulement. Les injonctions laconiques qui encadrent le poème : "Ajoutez une chaise à table "; «Enlevez donc la chaise », soulignent le refus d'introspection individuelle et collective mais aussi l'indifférence générale face aux souffrances et à la douleur juives.

Quelques mois plus tard, un débat littéraire est déclenché par la parution d'un livre édité sous la direction d'Adolf Endler et de Karl Mickel sous le titre : In diesem besseren Land. Gedichte der Deutschen Demokratischen Republik seit 1945 [Dans ce pays meilleur. Poèmes de la République Démocratique Allemande depuis 1945] ${ }^{23}$. L'organe du parti communiste est-allemand, le journal Neues Deutschland, s'attaque aux auteurs en leur reprochant de publier des poèmes qui auraient perdu le lien avec la réalité de la RDA et cite les noms des poètes Volker Braun, Karl Mickel, Bernd Jentzsch, Rainer Kirsch. La revue Forum, organe de presse de l'organisation des jeunesses socialistes, la « Freie Deutsche Jugend », propose alors aux écrivains de prendre la parole en répondant à trois questions qui s'attachent à cerner l'influence de la révolution industrielle et technique sur la poésie et le degré d'engagement de l'écrivain dans la société socialiste :

1. Le nouveau positionnement de l'homme dans la société socialiste, notamment par l'apport de la révolution technique, conduit-il à des évolutions dans le contenu et la structure de la poésie ?

2. À quelles conditions de la part de l'auteur et du lecteur la poésie peut-elle produire des effets dans notre société et quelles sont ces conditions?

3 . Devant quels grands problèmes créatifs vous trouvez-vous actuellement $?^{24}$

Günter Kunert prend le contre-pied du débat lancé en fustigeant la technique comme idéologie du progrès, véritable entreprise de mise à mort de l'homme par l'homme, et met en avant les dangers d'une

\footnotetext{
23 Adolf Endler, Karl Mickel, In diesem besseren Land. Gedichte der Deutschen Demokratischen Republik seit 1945, Berlin, Mitteldeutscher Verlag, 1966.

${ }^{24}$ Cité d'après E. Kasper, Zwischen Utopie und Apokalypse, op. cit., p. 58 : «1. Führt die neue Stellung des Menschen in der sozialistischen Gesellschaft, wie sie insbesondere durch die technische Revolution herbeigeführt wird, zu inhaltlichen und strukturellen Veränderungen der Lyrik ?; 2. Unter welchen Voraussetzungen seitens des Autors und des Lesers kann Lyrik in unserer Gesellschaft Wirkungen zeitigen, und wie können diese beschaffen sein ?; 3. Vor welchen hauptsächlichen Schaffensproblemen stehen Sie zur Zeit?».
} 
croyance aveugle dans la science et la technique. Pour Kunert, Auschwitz et la destruction des Juifs d'Europe et les bombes atomiques lancées sur Nagasaki et Hiroshima marquent le début de l'ère de la technique, ce sont les conséquences les plus fatales de l'accomplissement de la technique. Pour lui, il faudrait être bien naïf pour croire que l'évolution technique et industrielle signifie progrès humain, l'écrivain parlant au contraire d'un "abîme » toujours plus profond qui se creuserait entre le savoir-faire technique et l'existence humaine :

Ce qui m'apparait être la révolution technique la plus importante (et cela ne va pas tout à fait dans le sens de votre question), c'est la destruction massive d'hommes, la fin devenue possible de toute vie. Au début de l'ère technique, il y a Auschwitz, il y a Hiroshima, que je nomme ici d'un trait en référence à une technique socialement organisée. Je crois que seule une grande naïveté assimile la technique au progrès social et humain. Même si vous me taxez de scepticisme, terme devenu désormais courant: nous ne pouvons ignorer nos expériences et encore moins le monde dans lequel le fossé entre les possibilités techniques et la vie humaine ne cesse de grandir; voyage spatial d'un côté, misère crasse de l'autre: des contradictions d'ampleur globale prendront un jour des formes cosmiques. Fermer les yeux devant cette réalité signifie pour un écrivain dont l'objet est de regarder - et cela pas seulement depuis Goethe - de renoncer à l'un des rares principes de son métier. ${ }^{25}$

Et Günter Kunert illustre son propos en accompagnant son texte de deux poèmes, "Gewesene Größe der Eisenbahn » [Grandeur perdue du chemin de fer] et «Notizen in Kreide» [Notes à la craie] - dans ce second poème, l'auteur place au centre de la réflexion historique la destruction des Juifs d'Europe par les nazis, revient sur sa propre histoire familiale sur un ton de tristesse retenue et dénonce l'oubli des assassinés de la Shoah dans une Allemagne divisée dont les deux parties sont plus

\footnotetext{
${ }^{25}$ Günter Kunert in Forum 20 (1966/Heft 10), p. 23 : « Mir erscheint als bedeutendste technische Revolution (nicht ganz im Sinne Ihrer Frage) die Massenvernichtung von Menschen, das möglich gewordene Ende allen Lebens. Im Anfang des technischen Zeitalters steht Auschwitz, steht Hiroshima, die ich nur in Bezug auf gesellschaftlich organisierte Technik hier in einem Atemzug nenne. Ich glaube, nur noch große Naivität setzt Technik mit gesellschaftlich-humanitärem Fortschreiten gleich. Auch wenn Sie mich mit dem gerade gängigen Terminus Skeptiker abstempeln : Wir können unsere Erfahrungen nicht ignorieren, erst recht nicht die Welt, in der zwischen technischem Können und menschlichem Dasein die Kluft wächst ; Weltraumfahrt auf der einen, nackte Not auf der anderen Seite, Widersprüche globalen Ausmaßes nehmen eines Tages kosmische Formen an. Davor die Augen zu verschließen, bedeutet für einen Schriftsteller, der nicht erst seit Goethe zum Schauen bestellt ist, den Verzicht auf einen der wenigen Grundsätze seines Metiers ».
} 
soucieuses de développement économique que de retour sur un passé trop encombrant :

Notizen in Kreide

1 Eingerichtet auf dem Gestirn unseres Schmerzes

als Baracke. Aber fester denn jede Festung und dauernder. Ausgesetzt den bittersten Wettern. Ewiges Provisorium : ich.

2 Durchgangsstation

für Gefühle Gedanken Geträum.

Nach kurzem Aufenthalt

aufs immer neue leer und verödet

der Bahnhof meines angstvollen meines heillosen

Gebeins:

Aufgewirbelt

sinken zurück auf den versteinten Grund die Ascheflocken einst lebendig und verwandt demselben

der täglich meine Haut zu Märkten trägt und nicht fragt nach dem Preis.

3 Denen

sechs Millionen Tote eine Zahl ist

wie sechs Millionen Mark

wie sechs Millionen Sterne

wie sechs Millionen Konservenbüchsen

die Immergleichen

bekopfschütteln und verachselzucken

jenen den mein Name

entlarvt :

Der umherirrt

zwischen allen die keiner begraben kann

mit willfähriger Hand

kein Handlanger dieses Planeten auf diesem

Planeten:

Weil dessen Kruste aus Vergessen

noch zu dünn und

zu lebendig die fleißigen Töter

noch und wieder noch. 
[1. Installé sur l'astre / de notre douleur / comme une baraque. Mais plus solidement que chaque forteresse / et plus durablement. Exposé / aux intempéries les plus amères. Éternel provisoire: / Moi.; 2 Station de passage / pour sentiments pensées rêveries. / Après un court arrêt / encore et toujours vide et abandonnée / la gare de mes angoissés mes désespérés / Ossements : / Soulevés / retombent sur le sol bétonné / les flocons de cendre autrefois vivants et apparentés / à celui / qui quotidiennement commercialise ma dépouille / et ne demande pas son prix. ; 3 . À ceux / pour qui six millions de morts est un chiffre / comme six millions de Mark / comme six millions d'étoiles / comme six millions de boîtes de conserve / Les éternels mêmes / secouent la tête et haussent les épaules / devant celui que mon nom / démasque : / Celui qui erre / entre tous ceux que personne ne peut mettre en terre / d'une main complaisante / pas même le sbire de cette planète sur cette / planète : car sa croûte d'oubli est / encore trop mince et / trop vivants les tueurs zélés / encore et toujours].

Le « je » de ce poème se révèle bien éloigné de l'idéal socialiste ; « éternel provisoire », il se présente comme une «baraque », image qui d'emblée convoque les camps et leurs horreurs. Cette «baraque » est pourtant plus solide que toute «forteresse ». La deuxième strophe se fait personnelle, Kunert y évoque la peur après la perte des siens, le prix si lourd qu'il a fallu payer. La troisième strophe dit l'indifférence et l'oubli des assassinés de la Shoah : le chiffre de «six millions » est quatre fois martelé avec un sens de la provocation dûment assumé, comme pour faire prendre conscience de l'effroyable immensité du crime. Mais face à ce chiffre difficile à concevoir, le poème place l'indifférence et rappelle l'existence des « tueurs zélés » bien vivants. Le poème se termine sur un paradoxe, élément poétique qui caractérise à partir de cette période la production lyrique de Kunert, l'idée paradoxale selon laquelle, si les victimes juives sont présentes à la conscience des vivants, c'est parce que les assassins sont « trop vivants [...] encore et toujours » et ici.

\section{L'installation en RFA}

Quand Günter Kunert s'installe en RFA, nombreux sont ceux qui considèrent que le poète ainsi déraciné n'a plus rien à dire, le dissident n'aurait plus de matériau pour sa poésie ${ }^{26}$. Un débat orchestré par

\footnotetext{
26 Thomas Schmidt, Engagierte Artistik - Satire, Parodie und neoemblematische Verfahren im Werk Günter Kunerts, Würzburg, Ergon, 1998, p. 92 : « Kunerts Lyrik hat mit dem 'Platzwechsel' ihre wichtigste Funktion verloren. Einmal im Westen versteht man sie kaum mehr als stellvertretende Rede und erklärt statt dessen das 'Weltbild ' zur privaten Depression ».
} 
l'hebdomadaire hambourgeois Die Zeit illustre parfaitement cette perception occidentale ${ }^{27}$.

En 1982, Kunert présente aux lecteurs de la Zeit des poèmes d'auteurs contemporains et accompagne ces textes d'un commentaire introductif. La rédaction de l'hebdomadaire hambourgeois reçoit alors un vaste courrier dans lequel les lecteurs expriment leur mécontentement, reprochent à Kunert ses choix déprimants et s'interrogent sur l'existence d'une poésie égayante. Reprenant une critique faite à Kurt Tucholsky, grand publiciste allemand des années vingt et grand poète satirique, la Zeit lance le débat sous le titre « Wo bleibt das Positive ? » [Quand est-il du positif?].

Le numéro daté du 20 août 1982 publie un article de Hans-Jürgen Heise et la réponse de Günter Kunert. Dans ce premier article, fort réactif, Hans-Jürgen Heise reproche à la poésie moderne son pessimisme, parle des «Dichter der neuen Düsterkeit», des [poètes du nouveau sinistre] dont le retour à la rime classique ne ferait que souligner l'échec tant politique ou moral qu'esthétique. Ces poètes auraient pour fâcheuse habitude de généraliser leur état dépressif à un trait de l'époque actuelle. Et Heise de citer en conclusion le nom de Kunert comme exemple particulièrement révélateur de cette tendance catastrophiste de la littérature moderne.

La réponse de Günter Kunert est cinglante. Son article a pour titre : «Weltende oder private Misere? Wie blind sind die Propheten der neuen Fröhlichkeit?» [Fin du monde ou misère privée ? Les prophètes de la nouvelle gaieté sont-ils aveugles]. L'auteur y apostrophe la cécité des « prophètes de la nouvelle gaieté » et rejette toute approche utilitariste de la poésie. Il s'en prend également au reproche de subjectivisme fait à son encontre par Heise. Selon lui, le poète est un sismographe de son époque et propose au lecteur un espace de réflexion et de prise de conscience.

Dès 1976, Günter Kunert avait ainsi introduit l'image de l'épine pour qualifier l'écriture et la poésie, une épine à laquelle on se pique, qui nous fait mal et, par cette douleur, nous fait nous questionner sur nousmêmes », un « piquant qui blesse pour soigner ${ }^{28}$. Quinze ans plus tard,

\footnotetext{
27 Voir sur ce débat: Elke Kasper, «Untergangs- oder Überlebenskunst? Eine Lyrikdebatte und ihre Folgen ", dans Manfred Durzak / Helmuth Steinecke (éd.), Günter Kunert - Beiträge zu seinem Werk, München, Hanser, 1992, p. 102-119.

${ }^{28}$ Günter Kunert, «Ungunst und Hoffnung», dans Warum schreiben. Notizen zur Literatur. Essays, Berlin, Aufbau-Verlag, 1976, p. 268-269 : «ein Dorn, an dem man sich reisst und Schmerz verspürt und im Schmerz sich selber», "ein Stachel, der verwundet, um zu heilen ».
} 
le poète retrouve l'image de l'épine dans les conférences poétologiques qu'il tient à l'université de Francfort-sur-le-Main :

Et si le poème est capable d'avoir un effet, alors celui d'une épine qui s'enfonce dans la chair à moitié insensibilisée par le quotidien et les habitudes. L'enjeu du poème est de jeter le trouble. ${ }^{29}$

L'intention du poème est de bousculer, de bouleverser le lecteur dans son appréhension du monde $\mathrm{e}^{30}$, le poème est là pour extraire le lecteur de lui-même ${ }^{31}$. Loin d'être un pessimiste ou cette Cassandre de malheur que tant de critiques littéraires ont éreintée, Kunert se veut ainsi « un observateur lucide qui, contrairement aux autres, ne cherche pas à refouler ce qui va nous arriver $\gg{ }^{32}$.

C'est ainsi, par la force d'une poésie de l'engagement, d'une poésie qui dévoile et découvre, qui nous apostrophe et nous éprouve, que Kunert s'affirme poète allemand d'origine juive inscrit dans son temps.

\footnotetext{
${ }^{29}$ G. Kunert, Vor der Sintflut - das Gedicht als Arche Noah - Frankfurter Vorlesungen, op. cit., p. 51-52 : «Und wenn das Gedicht überhaupt Wirkung auszuüben vermag, dann nur die eines Stachels, der sich in das vom Alltag und den Gewohnheiten schon halb betäubte Fleisch bohrt. Seine Sache ist die ».

${ }^{30}$ Ibid., notamment p. 52: «Wenn der Leser, der das Gedicht mehr oder weniger intensiv nachvollzieht, sich um sein Leben betrogen fühlt, seiner Möglichkeiten und seiner Chancen beraubt; wenn das Gedicht sein Einverständnis mit der Welt erschüttert, dann hat es eine Leistung vollbracht, die für ein derart winziges Gebilde aus wenigen Zeilen gigantisch ist » [Si le lecteur, qui perçoit de manière plus ou moins intense le poème, se sent spolié de sa vie, de ses possibilités, de ses chances; si le poème bouleverse son appréhension du monde, alors le poème a réussi un véritable exploit pour une si petite chose qui tient en quelques lignes].

${ }^{31}$ G. Kunert, Warum schreiben, op. cit., p. 285 où Kunert dit «Entgröberung des Lesers ».

${ }^{32}$ Cf. Kunert dans une interview donnée en octobre 1990, reproduite dans : Maria Sonnenberg, Dissidenten? Texte und Dokumente zur DD - „Exil" Literatur, Volk und Wissen, Berlin, 1990: «Nur in den Augen von gänzlich haltlosen und grundlosen Optimisten bin ich ein Pessimist. Ich halte mich eher für einen nüchternen Betrachter, der nicht, wie die anderen, zu verdrängen sucht, was auf uns zukommt».
} 